\title{
Multi-objective Planning Model of Electric Vehicle Charging Station
}

\author{
Baoyi Wang ${ }^{\mathrm{a}, ~}$, Yongbo Zhou ${ }^{\mathrm{b}}$,Shaomin Zhang ${ }^{\mathrm{c}}$ \\ School of Control and Computer Engineering, North China Electric Power University, Baoding, \\ 071003, China \\ a.email:wangbaoyiqj@126.com,b.email:zyb19901108@126.com, \\ c. email:zhangshaomin@126.com
}

Keywords: Electric vehicle; charging station; queuing system; tabu-genetic algorithm

Abstract: Reasonable planning of electric vehicle charging station is of great significance for largescale use of electric vehicles. Based on the analysis of charging station construction requirements, a multi-objective planning model for charging station location problem considering users' satisfaction and operator benefits is constructed. From the point of view of users' satisfaction, consider the electric vehicle charging station's location problem with two parts, one is minimum of driving distance, the other is shortest time of waiting for charging. From the point of view of operator benefits, is the lowest cost of construction of charging station. Then, the improved tabu-genetic algorithm is proposed to solve this model. The test results show that the model proposed in this paper can effectively determine the locations and the service regions of the recharging stations, and the algorithm is quick and effective.

\section{Introduction}

With the development of electric vehicle technology, especially battery technology, and the strong support of policy in some countries, electric vehicles have developed rapidly in the past more than 10 years [1-2]. The document [3] proposed two stage geographical factors and the service radius of charging station location. According to the queuing theory, the model of charging facilities service system is established in document [4]. Document [5] considers the uneven distribution of electric vehicle demand in the planning area, and takes the minimum investment and operation costs as the goal. Document [6] uses dynamic traffic network theory to establish a charging station planning model. In document [7], the configuration method of charger based on queuing theory is put forward, and the optimization model of public charging station layout is established. Electric vehicle charging station is a city traffic public service facilities, its function is similar to the gas station [8-9].

Based on the above considerations, this paper draws lessons from the strategies of distributed power source location [10-11], and integrates the power facilities planning method [12]. From the point of view of users' satisfaction, consider the electric vehicle charging station's location problem with two parts, one is minimum of driving distance, the other is shortest time of waiting for charging. From the point of view of operator benefits, is the lowest cost of construction of charging station. Then, the improved tabu-genetic algorithm is proposed to solve this model. The test results 
show that the model proposed in this paper can effectively determine the locations and the service regions of the recharging stations, and the algorithm is quick and effective.

\section{Charging Station Queuing System}

In the promotion stage of electric vehicles, the charging station should consider the charging convenience of users first. With different fuel vehicles, electric vehicle charging time is longer, if there is not enough charge piles in the charging station for customer to service, it may cause queuing phenomena, and the charging station location is not only depends on the distance between users to the charging station, but also with greater importance about users' waiting time during the charging.

At present, most scholars directly refer to the queuing theory, the queuing model of charging station, few people collect user waiting time at the charging station, and it is difficult to collect such data in a large range, and so the user's waiting time is difficult to find an accurate probability distribution function to describe it. The general assumption of electric vehicle charging station arrival rate obeys the Poisson distribution, the user arrival time interval and the charging time satisfies the negative exponential distribution, this is the classic $\mathrm{M} / \mathrm{M} / \mathrm{k}$ single queue queuing system, multi server queuing system, $M / M / k$ users average waiting time is $\mathrm{W}_{\mathrm{q}}{ }^{\mathrm{M}}$

$$
\begin{aligned}
& W_{q}^{M}=\frac{(k \rho)^{k} \rho}{k !(1-\rho)^{2} \lambda} P_{0} \\
& P_{0}=\left[\sum_{i=1}^{k-1} \frac{1}{i !}\left(\frac{\lambda}{\mu}\right)^{i}+\frac{1}{k !} \frac{1}{1-\rho}\left(\frac{\lambda}{\mu}\right)^{k}\right]^{-1}
\end{aligned}
$$

The average queue length is:

$$
L_{q}^{M}=\frac{(k \rho)^{k} \rho}{k !(1-\rho)^{2}} P_{0}
$$

Among the formula, $\lambda$ is the user arrival rate, the average service efficiency of the single charging pile is $\mu$, and the number of charging piles in the charging station is $\mathrm{k}$.

The queuing system of electric vehicle charging station consists of two parts. From the user's point of view, always hope to reach the charging station immediately after the service, but also in the charging station service time as short as possible, if the number of stations charging more users in the whole process of waiting time is shorter; from the charging station operator's point of view, the lower using rates are waste of charging equipments, so it seems that it is impossible to increase the number of charging piles for improving service efficiency. Thus, it is necessary to consider the interests of both charging demand users and charging station operators, so that under certain reasonable conditions, the whole charging system service level can be optimal.

\section{Mathematical Model of Electric Vehicle Charging Station Planning Station}

\subsection{Basic Hypothesis and Objective Function}

In view of the fact that the situation is very complicated, in order to simplify the mathematical model, we make the following assumptions according to the problem description.

(1) Each point is inside user's living area, the number is equal to the number of electric vehicles.

(2) Every charging station user's arrival probability is certain, the remaining power is Gaussian distribution. 
(3) Each charging station is similar size.

(4) The type of vehicle or battery is same.

(5) Without the consideration of user's interests, they will choose the closest one for charging.

Assume that I represents a collection of demand points; $i \in I$, the number of demand point is $n$; the charging station candidate site is $\mathrm{j}, \mathrm{j} \in \mathrm{J}$, the number of it is $\mathrm{p}$; Xij is $0-1$ decision variables, if it is charging, the value is 1 , otherwise is 0 . yj is $0-1$ decision variables, if there will be a station, the value is 1 , otherwise is 0 . The objective function of this paper is as follows.

Function 1: the minimum distance between all users to the charging station

$$
\min Z_{1}=\sum \sum h_{i} d_{i j} x_{i j} y_{j}
$$

The $h_{i}$ is the user's number of demand point, $d_{i j}$ is the distance between point to charging station.

Function 2: the minimum sum of waiting time

Assuming that each car to arrive at the charging station interval follows $U$ ( $a, b)$ of uniform distribution. In general a charging station has more than 3 sets of charging pile; the charging stations generally have two kinds of methods, as shown on figure 1 .

For all candidate charging stations, the sum of waiting time is shortest, function 2 is as follows.

$$
\min Z_{2}=\sum \sum h_{i} d_{i j} x_{i j} T_{j m}
$$

Function 3: the minimum cost of charging station

$$
\min Z_{3}=\frac{1}{365} \sum_{j=1}^{p}\left[f\left(S_{j}\right) \frac{r_{0}\left(1+r_{0}\right)^{m}}{\left(1+r_{0}\right)^{m}-1}\right]
$$

Among them, $s_{j}$ is the number of charging piles at the charging station $j ; F$ is the cost of construction, $\mathrm{m}$ is the depreciation period of the charging station, and $\mathrm{r} 0$ is the discount rate. The cost function is based on the second order polynomial ${ }^{[13]}$ mentioned in the WANG H S' document.

$$
f\left(S_{j}\right)=100+10 S_{j}+3 S_{j}^{2}
$$

\subsection{Multi-objective Planning Model Constraints}

$$
\begin{aligned}
& \min Z_{1}=\sum \sum h_{i} d_{i j} x_{i j} y_{j} \\
& \min Z_{2}=\sum \sum h_{i} d_{i j} x_{i j} T_{j m} \\
& \min Z_{3}=\frac{1}{365} \sum_{j=1}^{p}\left[f\left(S_{j}\right) \frac{r_{0}\left(1+r_{0}\right)^{m}}{\left(1+r_{0}\right)^{m}-1}\right] \\
& \sum_{j \in J} x_{i j} y_{j} \geq 1 \\
& \sum_{j \in J} x_{i j}=1 \\
& x_{i j}, y_{j} \in\{0,1\}
\end{aligned}
$$

The objective function (8)(9)(10)has been already introduced, the constraint (11) indicates that all demand point will have corresponding charging stations; constraint (12) indicates that one point can only receive service in one charging station; constraint (13) is $0-1$ decision variables. 


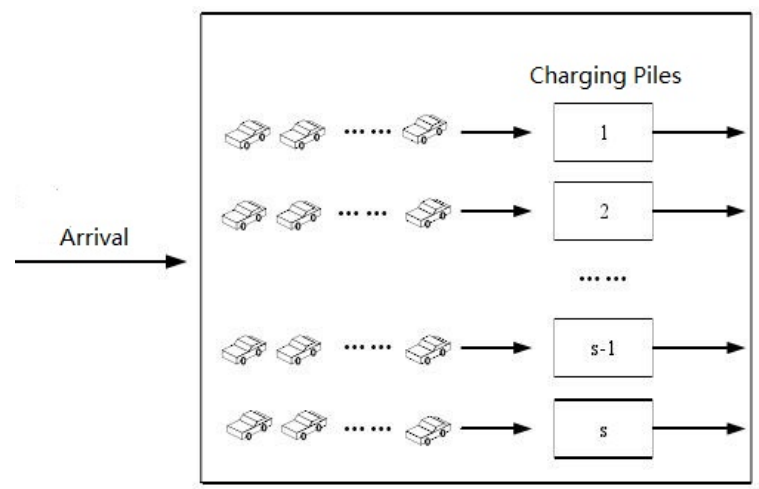

Fig.1 Multi queue mode

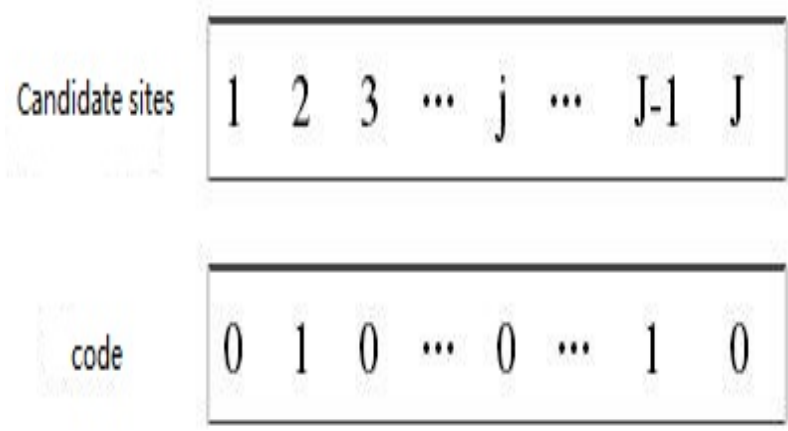

Fig. 2 coding method

\section{Tabu-genetic Algorithm for Model Solution}

\subsection{Chromosome Coding and Fitness Function}

The mechanism of genetic algorithm can not calculate the variables and objective function directly in the model, it needs encoding, so the genetic algorithm can identify the gene sequence data. Figure 2 is a typical chromosome code.

Fitness function is used to evaluate the quality of chromosome, which represents the survival ability of individuals in competition for survival. In this paper, the objective function Z1 and Z2 are converted into cost, the 14 form is the customer satisfaction cost, and the 15 form is the construction cost. The $\mathrm{c}_{\mathrm{ij}}$ is the unit distance cost of the demand point $\mathrm{i}$ to the charging station $\mathrm{j}$, and the $\mathrm{c}_{\mathrm{jm}}$ is the unit time cost of the users waiting in the charging station.

$$
\begin{aligned}
& f_{1}=\sum \sum c_{i j} h_{i} d_{i j} x_{i j} y_{j}+\sum \sum c_{j m} h_{i} d_{i j} x_{i j} T_{j m} \\
& f_{2}=\frac{1}{365} \sum_{j=1}^{p}\left[f\left(S_{j}\right) \frac{r_{0}\left(1+r_{0}\right)^{m}}{\left(1+r_{0}\right)^{m}-1}\right]
\end{aligned}
$$

Then, we standardize and process the multi-objective planning model of charging station, and construct the fitness function ${ }^{[14]}$ by using the weighted coefficient method.

$$
\begin{aligned}
& f_{1}^{\prime}=\frac{f_{1}-\min f_{1}}{\max f_{1}-\min f_{1}} \\
& f_{2}^{\prime}=\frac{f_{2}-\min f_{2}}{\max f_{2}-\min f_{2}}
\end{aligned}
$$

After that, we get the new fitness function.

$$
F=\omega_{1} f_{1}^{\prime}+\omega_{2} f_{2}^{\prime}
$$

The $\omega_{1}, \omega_{2}>0, F$ is the value of fitness function, the smaller the better.

\subsection{Cross Operator and Mutation Operator}

Tabu search to avoid circuit search through the tabu list and tabu criteria, we use tabu crossover operator and tabu mutation operator to improve crossover operator and mutation operator in traditional genetic algorithm, using a tabu list to record the current optimal solution and to seek information, comparing results and the results of the tabu list, excluding suboptimal solution, optimal solution record. 
Steps are as follows.

(1) Initialize the algorithm parameters, use paternal chromosome as the initial solution of taboo crossover and mutation operator.

(2) The current candidate set $\mathrm{N}(\mathrm{x})$ is generated, and the optimal solution $\mathrm{x}$ is selected from the candidate set.

(3) When the $F(x)$ is the optimal solution, the current optimal solution is updated. If not optimal, it is checked whether it is taboo, and the current solution and tabu list are updated.

(4) The tabu optimal solution is obtained.

\section{Case Study}

In order to verify the effectiveness of the proposed algorithm, we design demand points and some charging station in a region. All points generated from planar region of $[0,50] \mathrm{km} \times[0,50] \mathrm{km}$, the number of demand points is 30 . According to the principle of location of the charging station has 10 charging stations candidate. The electric vehicles arrive at the charging station interval follows a uniform distribution of $[10,30]$ minutes, the remaining power of electric vehicles when arrived at the charging station follows Gaussian distribution, $\mathrm{N}\left(0.15,0.1^{2}\right)^{[15]}$.

The data of electric vehicles using BYD E6 model, its energy can last for 300 kilometres, fast charging 20 minutes could be filled with 98 percent; each hundred kilometres need $21.5 \mathrm{kw} \cdot \mathrm{h}$, electric vehicle charging costs 1 Yuan / $\mathrm{kw} \cdot \mathrm{h}$; time loss cost is 10 Yuan / hour; charging station depreciation $\mathrm{m}=20$; discount rate $\mathrm{r}_{0}=0.08$; the objective function weights $\omega_{1}$ and $\omega_{2}$ are all 0.5. In the genetic algorithm, the crossover rate is 0.85 , the mutation rate is 0.45 , and the reproduction algebra is 300 .

The simulation experiments are carried out on MATLAB 2016a by using tabu-genetic algorithm, and the results of charging station planning are obtained. From 10 candidate charging stations, 4 of them are selected as service stations, and the specific results are shown in table 1. According to the result, a diagram is drawn, as shown on figure 3.

Table 1 Charging station location and service area

\begin{tabular}{|c|c|}
\hline location & service area \\
\hline 2 & $2,3,4,10,14,17,18,22,23,24,26$ \\
\hline 5 & $1,6,11,13,29,30$ \\
\hline 6 & $7,8,9,12,15,16,20,27,28$ \\
\hline 10 & $5,19,21,25$ \\
\hline
\end{tabular}

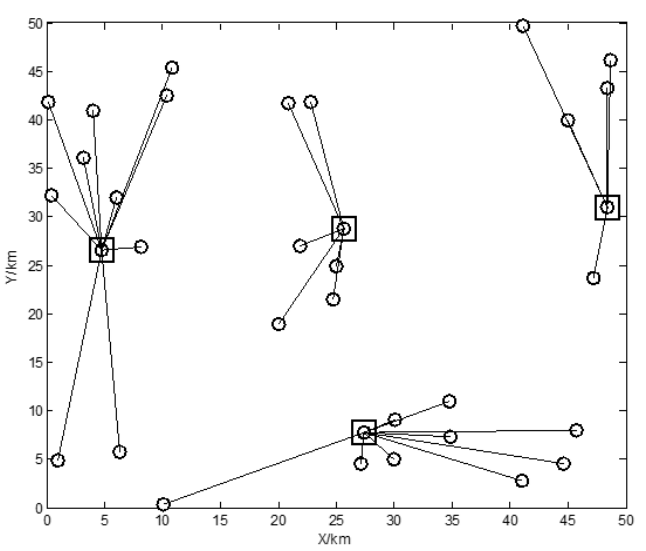

Fig.3 Charging station location and service area 


\section{Conclusion}

In this paper, we construct a planning model for charging station location problem considering users' satisfaction and operator benefits. From the point of view of users' satisfaction, consider the electric vehicle charging station's location problem with two parts, one is minimum of driving distance, the other is shortest time of waiting for charging. From the point of view of operator benefits, is the lowest cost of construction of charging station. Then, the improved tabu-genetic algorithm is proposed to solve this model. The test results show that the model proposed in this paper can effectively determine the locations and the service regions of the recharging stations, and the algorithm is quick and effective.

It should be pointed out that this study assumes that the owner of the electric vehicle has no preference, and is charged according to the shortest path. In reality, the owner may not be able to choose the shortest path, sometimes the path may have traffic jam and unable to drive, how to properly consider these practical factors will be the next stage of the study.

\section{References}

[1] SCHNEIDER K,GERKENSMEYER C, KINENERMEYER M, et al. Impact assessment of plug-in hybrid vehicles in Pacific Northwest distribution systems[C]/Proceedings of IEEE Power Engineering Society General Meeting, July 20-24, 2008, Pittsburgh, PA, USA:1-6.

[2] XU Fan, YU Guoqin, GU Linfeng, et al. Tentative analysis of layout of electrical vehicle charging stations [J]. East China Electric Power, 2009, 37(10): 1678-1682.

[3] LIU Zhipeng, WEN Fushuan, XUE Yusheng, et al. Optimal sitting and sizing of electric vehicle charging stations [J]. Automation of Electric Power Systems.12012, 36(3): 54-59.

[4] LI Ruqi, SU Haoyi. Optimal allocation of charging facilities for electric vehicles based on queuing theory [J]. Automation of Electric Power Systems, 2011, 35(14): 58-61.

[5] XIONG Hu, XIANG Tieyuan, RONG Xin, et al. Optimal allocation of electric vehicle battery swap stations [J]. Electric Power Automation Equipment, 2012, 32(9): 1-6.

[6] REN Yulong, SHI Lefeng, ZHANG Qian, et al. Optimal distribution and scale of charging stations for electric vehicles [J]. Automation of Electric Power Systems, 2011, 35(14): 53-57.

[7] XIONG Hu, XIANG Tieyuan, ZHU Yonggang, et al. Electric vehicle public charging stations location optimal planning [J]. Automation of Electric Power Systems, 2012, 36(23): 65-70.

[8] SONG Yahui. Research on the Layout Planning of Electric Vehicle Charging Station in the City [D]. Beijing Jiaotong University, 2011.

[9] CUI Jing. Study on the Layout of Gas Stations in Our Country's City [D]. China University of Petroleum, 2008

[10] LIU Zhipeng, WEN Fushuan, XUE Yusheng, et al. optimal sitting and sizing of distributed generators considering plug-in electric vehicles [J]. Automation of Electric Power Systems, 2011, 35(18): 11-16.

[11] HUANG D Q, MITHULANANTHAN N, BANSAL R C. Analytical expressions for DG allocation in primary distribution networks [J]. IEEE Trans on Energy Conversion, 2010, 25(3):814-820.

[12] WANG Chengshan, :LIU Tao, XUE Yusheng. Substation location and sizing based on hybrid genetic algorithm [J]. Automation of Electric Power Systems, 2006, 30(6): 30-34.

[13] Wang H, Huang Q, Zhang C, et al. A novel approach for the layout of electric vehicle charging station [C] // International Conference on Apperceiving Computing and Intelligence Analysis (ICACIA 2010). Chengdu, China: IEEE, 2010:64-67.

[14] Zhao J, Zhou H, Liang C, et al. Hybrid optimization based on genetic-tabu search for JLSP[J]. Systems Engineering and Electronics, 2012,34(4):833-838.

[15] CHEN Lianfu. Research on the Planning and Layout of Electric Taxi Charging Station [D]. Beijing Jiaotong University, 2015. 